\title{
Optimal roughening of convex bodies
}

\author{
Alexander Plakhov*
}

\begin{abstract}
A body moves in a rarefied medium composed of point particles at rest. The particles make elastic reflections when colliding with the body surface, and do not interact with each other. We consider a generalization of Newton's minimal resistance problem: given two bounded convex bodies $C_{1}$ and $C_{2}$ such that $C_{1} \subset C_{2} \subset \mathbb{R}^{3}$ and $\partial C_{1} \cap \partial C_{2}=\emptyset$, minimize the resistance in the class of connected bodies $B$ such that $C_{1} \subset B \subset C_{2}$. We prove that the infimum of resistance is zero; that is, there exist "almost perfectly streamlined" bodies.
\end{abstract}

Mathematics subject classifications: 37D50, 49Q10

Key words and phrases: Billiards, shape optimization, problems of minimal resistance, Newtonian aerodynamics, rough surface.

\section{Introduction}

Consider a solid body moving through a rarefied medium. The medium consists of point particles, initially (prior to collisions with the body) at rest. Each particle can make one or several collisions with the body surface; we assume that these collisions are perfectly elastic and the particles do not interact with each other. As a result of the collisions, a resistance force is created that acts on the body and slows down its motion. The problem of minimal resistance consists in finding the body, from a given class of bodies, that experiences the smallest possible resistance force (provided that the velocity of the body and the medium density are fixed).

The first person to state and solve such a problem was I. Newton; in [13] he found the body of minimal resistance in the class of convex axisymmetric bodies inscribed in a fixed cylinder, where the symmetry axis of the body coincides with the cylinder axis and is parallel to the direction of motion of the body. The solution in the case where the cylinder diameter is equal to its height is shown in Fig. 1.

*University of Aveiro, Department of Mathematics, Aveiro 3810-193, Portugal 


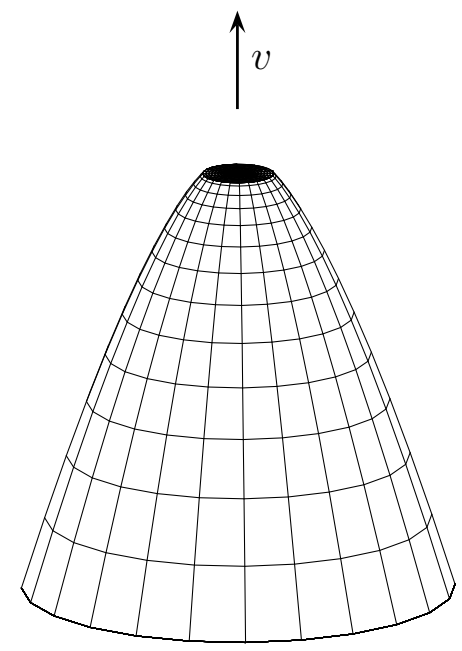

Figure 1: Newton's body of minimal resistance. The vector $v$ indicates the direction of motion of the body.

In 1990s it was discovered that the resistance can be further decreased by breaking the axial symmetry of the body. More precisely, in the wider class of convex (not necessarily symmetric) bodies inscribed in the same cylinder, the minimizer exists and does not coincide with Newton's optimal body [7, 5, 3]. This important discovery gave rise to many interesting works on the minimal resistance problem in various classes of bodies, both convex and nonconvex $[6,1,11,12,8,9,10,2,4,14,15,16]$.

These problems can be naturally interpreted in terms of space aerodynamics. Suppose we are designing a spacecraft for a long galactic travel. The problem is to find the best shape for the spacecraft, so as to minimize the velocity loss when traveling through huge interstellar clouds on the way. The choice of an admissible class of shapes may be dictated by technological restrictions.

In this paper we provide a slightly different look at the problem. Suppose we are traveling in a spaceship $C_{2} \subset \mathbb{R}^{3}$, which is a bounded convex set. The inner space of the spaceship coincides with another convex set $C_{1} \subset C_{2}$ (see Fig. 2). The spaceship body is then $C_{2} \backslash C_{1}$; it is natural to require that $\partial C_{1} \cap \partial C_{2}=\emptyset$ (this means that the thickness of the spacecraft body is everywhere positive).

We are going to process the metallic body of the spaceship aiming to minimize the velocity slowdown when going through space clouds. The processing may result in making dimples, hollows, grooves, etc on the spaceship surface. In general, we assume that any body $B$ satisfying the inclusions $C_{1} \subset B \subset C_{2}$ can be obtained by processing. We put the following

Question: Given the convex bodies $C_{1}$ and $C_{2}$, the spaceship velocity $v$ and the cloud density, what is the minimum resistance of the resulting body $B$ ?

In some cases the resistance of the original body $C_{2}$ can be easily decreased just by 


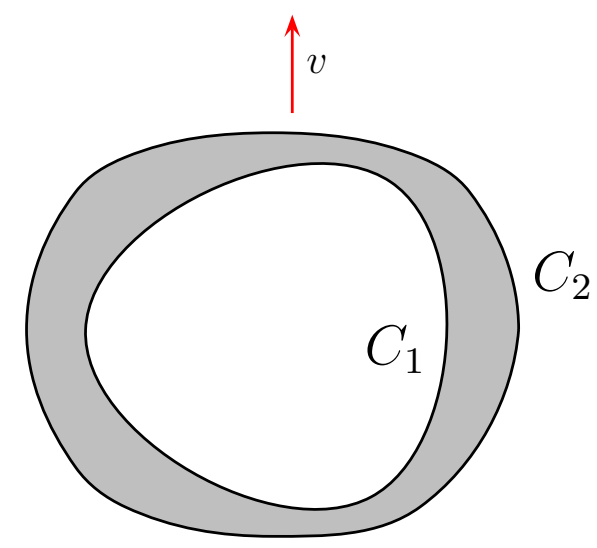

Figure 2: The spaceship. The direction of motion is indicated by $v$.

making dimples. Indeed, let the direction of $v$ be vertical and consider a region $\mathcal{U} \subset \partial C_{2}$ in the upper part of the surface $\partial C_{2}$ whose inclination relative to the horizontal plane is less than $30^{\circ}$. (We assume that $\mathcal{U}$ is not empty.) Then make several conical dimples in this region, with the inclination of the cone surface being exactly $30^{0}$ (see Fig. 3 (b)).

The resistance of the resulting body $B$ is smaller than that of the original body $C_{2}$. Indeed, in the reference system connected with the body we observe a parallel flow of particles falling vertically down. A particle hitting $C_{2}$ in the region $\mathcal{U}$ is reflected at an angle smaller than $60^{\circ}$ relative to the vertical (see Fig. 3 (a)). On the contrary, a particle hitting $B$ in a conical dimple will be reflected exactly at the angle $60^{0}$ (see Fig. 3 (b)). Therefore the momentum transmitted by the particle to the body is smaller in the latter case than in the former one, and summing up all the transmitted momenta, we get that the resistance of $B$ is smaller than that of $C_{2}$.

In a similar way, the resistance of Newton's optimal body can be decreased by making dimples on its front (flat) surface. This observation was first made by Buttazzo and Kawohl in [7]. The techniques of making dimples and grooves were further developed by Comte and Lachand-Robert in $[8,9,10]$ when studying generalizations of Newton's problem in classes of nonconvex bodies.

The answer to our question is surprising: the resistance of the resulting body can be made arbitrarily small. That is, by processing the surface of our spaceship, one can make it almost perfectly streamlined! This paper is mainly devoted to the proof of this statement. Note that this result was announced, with a brief outline of the proof, in [16].

The paper is organized as follows. In Section 2 the basic mathematical definitions are given and the main results of the paper, Theorems 1 and 2, are stated. In Section 3 an auxiliary construction, a channel, is described and studied in detail. This construction is then used in Sections 4 and 5 when proving Theorems 2 and 1. 

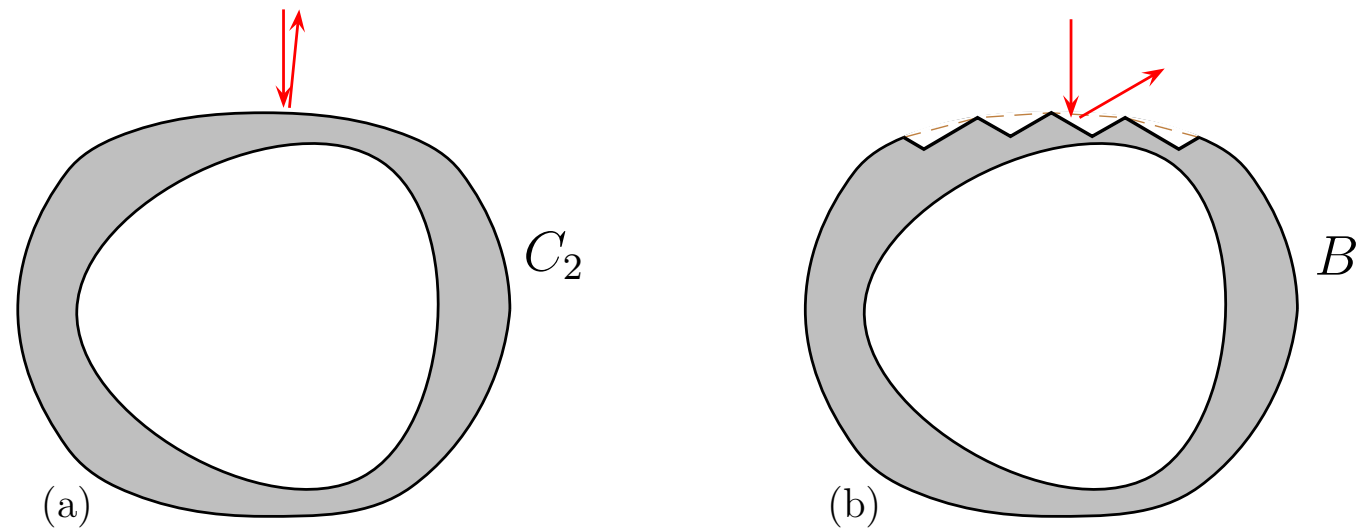

Figure 3: The original spaceship $C_{2}$ (a) and the spaceship $B$ with dimples (b).

\section{Definitions and formulation of the results}

Definition 1. A bounded set $B \subset \mathbb{R}^{d}$ with piecewise smooth boundary is called $a$ body.

Consider the billiard in $\mathbb{R}^{d} \backslash B$. Suppose that a billiard particle initially moves freely according to $x(t)=x+v t$, then makes a finite number of reflections (maybe none) from $\partial B$, and finally moves freely again according to $x(t)=x^{+}+v^{+} t$. This description defines the mapping

$$
(x, v) \mapsto\left(x^{+}=x_{B}^{+}(x, v), v^{+}=v_{B}^{+}(x, v)\right),
$$

which is defined on a full measure subset of $\mathbb{R}^{d} \times S^{d-1}$, takes values in $\mathbb{R}^{d} \times S^{d-1}$ and is measure preserving. Here the measure is the product of Lebesgue measures on $\mathbb{R}^{d}$ and $S^{d-1}$. The mapping (1) is called scattering mapping.

Definition 2. If the restriction $\left.v_{B}^{+}\right\rfloor_{\substack{v=v_{0} \\ x \in v_{0}^{\perp}}}$ of the function $v_{B}^{+}$to the subset $v=v_{0}, x \in v_{0}^{\perp}$ is measurable and defined almost everywhere with respect to Lebesgue measure on $v_{0}^{\perp}$, then $v_{0}$ is called a regular direction for the body $B$.

The set of regular directions for any body $B$ has full measure in $S^{d-1}$. An example of a non-regular direction for a two-dimensional body $B$ is provided in Fig. 4. A part of the boundary $\partial B$ is an arc of a parabola with focus at a singular point $F$ of the boundary. The particles that initially move with the velocity $v$ parallel to the parabola axis, after reflecting from the arc hit the body at the point $F$, and their further motion is not defined. Therefore the direction $v$ is not regular for $B$.

Suppose the direction $v_{0}$ is regular for $B$. Note that the function $v-v_{B}^{+}(x, v)$ is bounded and compactly supported on the subset $v=v_{0}, x \in v_{0}^{\perp}$, and therefore, is integrable with respect to Lebesgue measure in $v_{0}^{\perp}$. 


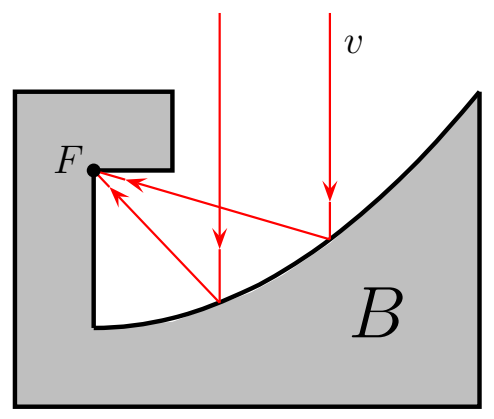

Figure 4: $v$ is the non-regular direction for the body $B$.

Definition 3. Let $v \in S^{d-1}$ be a regular direction for $B$. Then the integral

$$
R_{v}(B)=\int_{v^{\perp}}\left(v-v_{B}^{+}(x, v)\right) d x
$$

is called the resistance of the body $B$ in the direction $v$.

Remark 1. This definition has a direct physical meaning. An incident particle with the initial velocity $v$ transmits the momentum $\mu\left(v-v^{+}\right)$to the body, where $v^{+}=v_{B}^{+}(x, v)$ is the velocity of the reflected particle and $\mu$ is its mass. The resistance is the sum of all momenta transmitted to the body in a unit time. The summation amounts to integrating over $v^{\perp}$; as a result one gets $\rho R_{v}(B)$, where $\rho$ is the medium density.

In what follows we restrict our consideration to those bodies $B$ for which the chosen direction $v$ is regular, and therefore, the integral $R_{v}(B)$ is defined.

Consider the three-dimensional case where $v \in S^{2}, C_{1}$ and $C_{2}$ are bounded convex bodies, $C_{1} \subset C_{2} \subset \mathbb{R}^{3}$ and $\partial C_{1} \cap \partial C_{2}=\emptyset$. The main result of the paper reads as follows.

Theorem 1. In the three-dimensional case holds

$$
\inf \left\{\left|R_{v}(B)\right|: C_{1} \subset B \subset C_{2}, B \text { connected }\right\}=0 .
$$

The proof of this theorem is based on the following auxiliary two-dimensional result. Let $C_{1}$ and $C_{2}$ be bounded convex bodies, $C_{1} \subset C_{2} \subset \mathbb{R}^{2}, \partial C_{1} \cap \partial C_{2}=\emptyset$ and $v \in S^{1}$.

Theorem 2. In the two-dimensional case holds

$$
\inf \left\{\left|R_{v}(B)\right|: C_{1} \subset B \subset C_{2}\right\}=0 .
$$

Remark 2. Notice that the two-dimensional theorem is weaker than the threedimensional one, since the infimum is taken over the wider class of (generally) disconnected bodies. On the contrary, the infimum over connected bodies is always positive in two dimensions. 
The following plausible conjecture is intended to further elucidate the difference between the two-dimensional and three-dimensional case.

Definition 4. Let $C \subset \mathbb{R}^{d}$ be a bounded convex body and $v \in S^{d-1}$. The value

$$
\mathfrak{R}_{v}(C):=\sup _{C_{1}} \inf \left\{\left|R_{v}(B)\right|: C_{1} \subset B \subset C, B \text { connected }\right\},
$$

where the supremum is taken over all convex bodies $C_{1}$ such that $C_{1} \subset C$ and $\partial C_{1} \cap \partial C_{2}=$ $\emptyset$, is called the minimal resistance of bodies obtained by roughening $C$.

Conjecture 1. (a) For any $v \in S^{2}$ and any convex $C \subset \mathbb{R}^{3}$ holds

$$
\mathfrak{R}_{v}(C)=0 .
$$

(b) For any $v \in S^{1}$ and any convex $C \subset \mathbb{R}^{2}$ holds

$$
1 / 4<\frac{\mathfrak{R}_{v}(C)}{\left|R_{v}(C)\right|} \leq 1 / 2
$$

The statement (a) of this conjecture is a direct consequence of Theorem 1, but the statement (b) is not proved yet. Note, however, that in the particular case, where $C$ is symmetric with respect to an axis parallel to $v$, the double inequality (2) can be easily derived from the proof of Theorem 2 of the paper [16].

More information on billiard scattering by rough bodies and related minimal resistance problems can be found in [14] and [16].

Let us state one more plausible conjecture.

For a set $D \subset \mathbb{R}^{d}$ and a point $x \in \mathbb{R}^{d}$, put $\operatorname{dist}(x, D)=\inf \{|x-y|: y \in D\}$, and for $\varepsilon>0$ denote by $\mathcal{U}_{\varepsilon}(D)$ the $\varepsilon$-neighborhood of $D$,

$$
\mathcal{U}_{\varepsilon}(D)=\left\{x \in \mathbb{R}^{d}: \operatorname{dist}(x, D)<\varepsilon\right\} .
$$

Conjecture 2. (a) For any connected bounded set $D \subset \mathbb{R}^{3}$ and any $v \in S^{2}$ and $\varepsilon>0$,

$\inf \left\{\left|R_{v}(B)\right|: B\right.$ is connected and $\left.D \subset B \subset \mathcal{U}_{\varepsilon}(D)\right\}=0$.

(b) For any bounded set $D \subset \mathbb{R}^{2}$ and any $v \in S^{1}$ and $\varepsilon>0$,

$$
\inf \left\{\left|R_{v}(B)\right|: D \subset B \subset \mathcal{U}_{\varepsilon}(D)\right\}=0 .
$$

The claim of Conjecture 2, part (a), is stronger than that of Theorem 1, since the convex bodies $C_{1}$ and $C_{2}$ are replaced here with connected sets $D$ and $\mathcal{U}_{\varepsilon}(D)$. We are sure it is also true and can be proved using the idea of channel system (see the next section), but the proof will be much more cumbersome than that of Theorem 1 . The part (b) of Conjecture 2 is analogous to Theorem 2 and is valid in two dimensions, with the resulting bodies of small resistance being disconnected. 


\section{Preliminary constructions}

Here we explain the basic two-dimensional construction which will be used in the proofs of Theorems 2 and 1. The main idea of these proofs consists in making a large number of "diversion channels" penetrating the body near its boundary. Each channel is the union of three sets: a front channel, a tube, and a rear funnel. The front funnel is turned to the flow, and the rear one, to the opposite direction. Each particle of the flow gets into the front funnel of a channel, then moves through the channel along the body boundary, and escales through the rear funnel, and its final velocity only slightly differs from the velocity of incidence of the flow.

Introduce the coordinates $x_{1}, x_{2}$ on the plane such that $v=(0,-1)$, the $x_{1}$-axis being considered to be horizontal, and the $x_{2}$-axis, vertical. Fix the parameter $0<\varepsilon<1$. The front and rear $\varepsilon$-funnels $V_{ \pm}$are the trapezoids $\left|x_{1}\right| \leq \varepsilon\left|x_{2}\right|, \varepsilon^{2} \leq \pm x_{2} \leq \varepsilon$, respectively. The point $\left(0, \pm \varepsilon^{2}\right)$ is called the vertex of the corresponding funnel. The front and rear sides of the front funnel are, respectively, its larger and smaller bases, that is, the segments $\left\{\left|x_{1}\right| \leq \varepsilon^{2}, x_{2}=\varepsilon\right\}$ and $\left\{\left|x_{1}\right| \leq \varepsilon^{3}, x_{2}=\varepsilon^{2}\right\}$. On the contrary, the front and rear sides of the rear funnel are its smaller and larger bases, that is, the segments $\left\{\left|x_{1}\right| \leq \varepsilon^{3}, x_{2}=-\varepsilon^{2}\right\}$ and $\left\{\left|x_{1}\right| \leq \varepsilon^{2}, x_{2}=-\varepsilon\right\}$. A parallel translation of the front (rear) funnel is also called a front (rear) funnel. See Fig. 5 (a).

An $\varepsilon$-tube is a finite sequence of figures: rectangles and circle sectors. These figures are called elements of the tube. The rectangles are vertically or horizontally oriented; they are called $v$-and $h$-rectangles, respectively. In a $v$-rectangle, one of the horizontal (upper and lower) sides is considered to be the front side, and the other horizontal side is the rear one. Their length equals $2 \varepsilon^{3}$. In a $h$-rectangle, the length of the vertical (left and right) sides equals $2 \varepsilon^{3}$; one of these sides is the front one, and the other side is the rear one. Each circle sector has the angular size $90^{\circ}$; it is a quarter of a circle of the radius $2 \varepsilon^{3}$. One of the radii bounding the sector is vertical, and the other one is horizontal; one of these radii is called the front one, and the other, the rear one. In the sequence of figures forming the tube, rectangles and circle sectors alternate; the first and the last figure are $v$-rectangles, the upper side of the first rectangle is the front one, and the lower side of the last rectangle is the rear one; see Fig. 5 (a). Further, in the subsequence composed of rectangles the $v$ - and $h$-rectangles alternate. Finally, in the sequence of figures (rectangles and circle sectors) forming the tube, the rear side of the preceding figure coincides with the front side of the subsequent figure, and there are no other points of pairwise intersection of the figures.

It may happen, in particular, that the tube is a single $v$-rectangle; in this case its upper side is the front one, the lower side, the rear one, and the length of these sides is $2 \varepsilon^{3}$.

Definition 5. An $\varepsilon$-channel is the union of a front $\varepsilon$-funnel, an $\varepsilon$-tube, and a rear $\varepsilon$-funnel,

$$
K=V_{+} \cup T \cup V_{-} \subset \mathbb{R}_{\left\{x_{1}, x_{2}\right\}}^{2},
$$




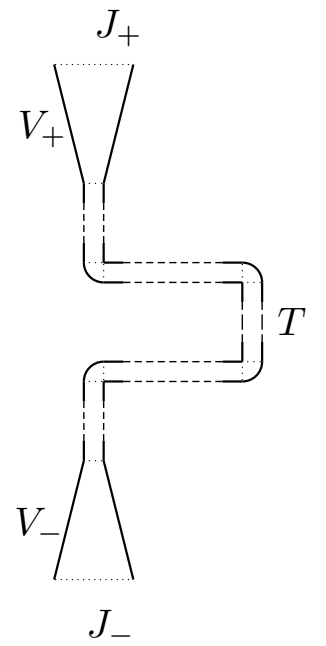

(a) Channel.

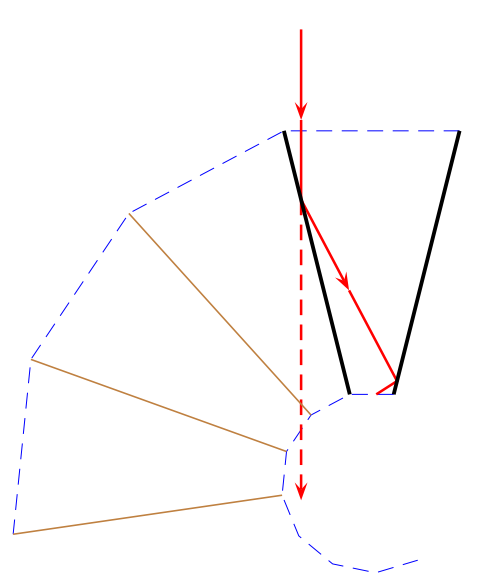

(b) Motion of a particle in the front funnel.

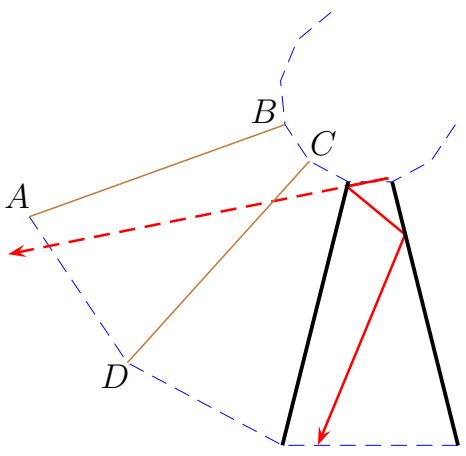

(c) Motion of a particle in the rear funnel.

Figure 5: Dynamics of a particle in a channel.

satisfying the following conditions: the rear side of the front funnel coincides with the front side of the tube, the rear side of the tube coincides with the front side of the rear funnel, and there are no other points of pairwise intersection for these figures. The front part of the front funnel is called the front side of the channel (denoted by $J_{+}$in Fig. 5 (a)), and the rear side of the rear funnel is called the rear side of the channel (denoted by $J_{-}$in Fig. $5(\mathrm{a})$ ). The rest of the channel boundary is called the lateral boundary of the channel.

Lemma 1. Consider the billiard in an $\varepsilon$-channel. If a particle starts the motion with the velocity $v=(0,-1)$ at a point of the front side of the channel, then after making a finite number of reflections from the lateral boundary, it finally gets into a point of the rear side of the channel with the velocity $v+O(\varepsilon), \varepsilon \rightarrow 0$. Here $O(\varepsilon)$ is uniform with respect to all $\varepsilon$-channels and all initial positions.

Proof. First we prove that the particle, after a finite number of reflections from the lateral boundary, gets into the rear side of the channel (and not into its front side). The proof of this statement is inductive. Namely, for each figure forming the channel (trapezoid, rectangle, circle sector) we will prove the following: if the particle gets into the figure through its front side, then after a while it will leave the figure through its rear side.

The motion in a rectangle is unidirectional, from the front to the rear side; this is obvious. Further, notice that when moving in a circle, the angular coordinate of the particle changes monotonically. This implies that if the particle intersects the front radius of a sector, then after several (maybe none) reflections from the arc it will intersect the 
rear radius.

It remains to consider the motion in the funnels. The particle starts moving vertically down from the front side of the front funnel (that is, from the larger side of the trapezoid). Apply the method of unfolding of the trajectory; see Fig. 5 (b). In a convenient reference system the trapezoid takes the form $\left|x_{1}\right| \leq \varepsilon x_{2}, \varepsilon^{2} \leq x_{2} \leq \varepsilon$. The unfolded trajectory is a vertical line at a distance less than $\varepsilon^{2}$ from the origin; therefore it intersects the circle $\operatorname{Ball}_{\varepsilon^{2}}(0)$. On the other hand, the sequence of images of the smaller side of the trapezoid under the unfolding forms a broken line winding around the origin and touching the same circle. (Notice that this broken line is contained in the larger circle $\mathrm{Ball}_{\varepsilon^{2} \sqrt{1+\varepsilon^{2}}}(0)$; we will use it later.) Hence the unfolded trajectory intersects the broken line; this means that the original trajectory, after several reflections from the lateral sides of the trapezoid, will intersect its smaller side.

Finally, when considering the motion in the rear funnel we again use the unfolding method. This time we unfold the final part of the trajectory starting from the point of intersection with the front side of the funnel (that is, the smaller base of the trapezoid; see Fig. $5(\mathrm{c}))$. The unfolded trajectory intersects one of the images, under the unfolding, of the larger base of the trapezoid; this image is $A D$ in the figure. This means that the particle, after several reflections from the lateral sides of the trapezoid, finally reaches the rear side of the channel. Using Fig. 5 (c), one gets an estimate for the particle velocity at the point of intersection with the rear side of the funnel. The angle the velocity vector forms with the vertical is obviously smaller than the largest angle formed by the symmetry axis of $A B C D$ with the tangent lines from $A$ to the circle $\operatorname{Ball}_{\varepsilon^{2} \sqrt{1+\varepsilon^{2}}}(0)$. The latter value equals $\arctan \varepsilon+\arcsin \varepsilon$. Thus, the difference between the initial and final velocities, $v$ and $v^{+}$, of a particle in an $\varepsilon$-channel can be estimated from above as follows:

$$
\left|v-v^{+}\right| \leq 4 \sin ^{2}((\arctan \varepsilon+\arcsin \varepsilon) / 2)=O(\varepsilon) .
$$

The figure 6 shows how the channel system may look like in the case where $C_{1}$ and $C_{2}$ are concentric squares. A body of small resistance is obtained by removing the channels from the larger square $C_{2}$.

The construction of the channel system in the general case is more complicated. We will start with a method of constructing a special $\varepsilon$-channel which will be used later on in this section. Consider two rectangles $\Pi^{+}$and $\Pi^{-}$with the horizontal sides of length $2 \varepsilon^{2}$ and vertical sides of length $\varepsilon-\varepsilon^{2}$, and let $A^{+}$be the midpoint of the lower side of $\Pi^{+}$, and $A^{-}$, the midpoint of the upper side of $\Pi^{-}$. A broken line joining the points $A^{+}$and $A^{-}$and satisfying the conditions stated below in this paragraph will be called an $\varepsilon$-axis, and these points will be called the front and rear endpoints of the axis. The broken line consists of a finite number of vertical and horizontal segments. The initial and final segments are vertical ones of lengths more or equal than $\varepsilon^{3}$, and the lengths of the other segments are more or equal than $2 \varepsilon^{3}$. The broken line does not have points of 


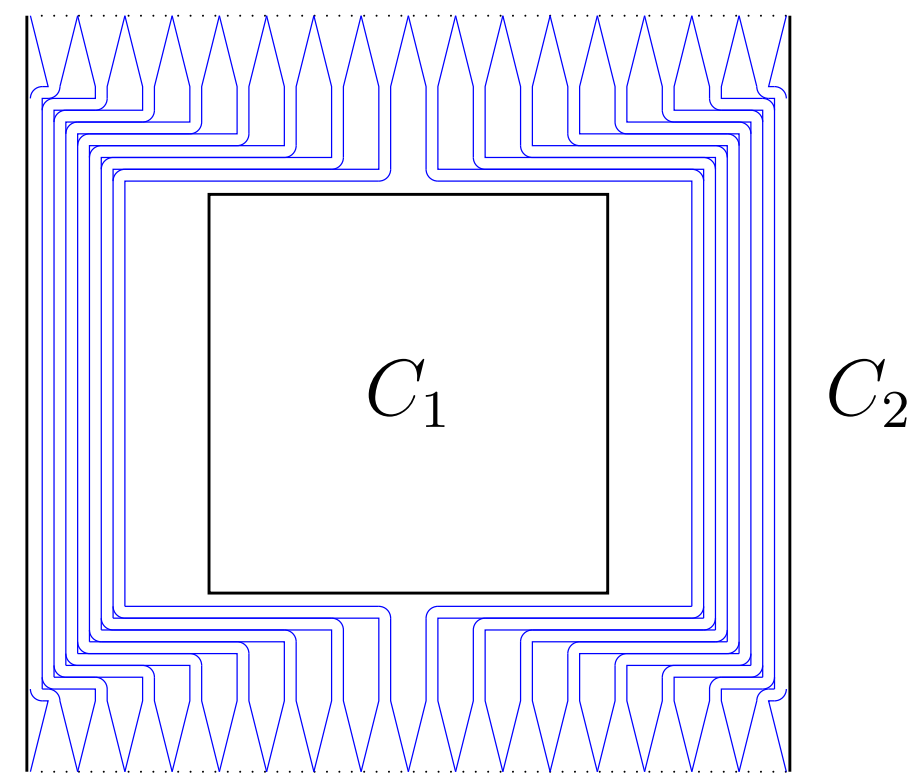

Figure 6: Two concentric squares with a built-in channel system.

self-intersection and does not have points of intersection with $\Pi^{+}$and $\Pi^{-}$others than the endpoints $A^{+}$and $A^{-}$. The endpoints of the segments, except for $A^{+}$and $A^{-}$, are called vertices. Thus, both the initial and final segments have only one vertex, and the other segments have two vertices. A shortened segment of the broken line, with (one or two) segments of length $\varepsilon^{3}$ adjacent to its vertices taken off, is called a reduced segment. A reduced segment may be a true segment, and may be a single point. The union of the rectangles $\Pi^{+}$and $\Pi^{-}$and the $\varepsilon$-axis is called an $\varepsilon$-contour; see Fig. 7 (a).

Now suppose we have an $\varepsilon$-contour. To each reduced segment of the $\varepsilon$-axis assign the rectangle of width $2 \varepsilon^{3}$ such that the segment is a midline of the rectangle and divides it into two rectangles of width $\varepsilon^{3}$; see Fig. 7 (b). In the degenerated case, where the reduced segment is a point, the assigned rectangle is a segment of length $2 \varepsilon^{3}$. To each vertex assign the circle sector of radius $2 \varepsilon^{3}$ such that the two radii bounding the sector coincide with sides of the rectangles assigned to the adjacent reduced segments. Finally, to the rectangles $\Pi^{+}$and $\Pi^{-}$assign the inscribed trapezoids $V^{+}$and $V^{-}$such that the midpoints of two sides of these rectangles, $A^{+}$and $A^{-}$, are also midpoints of smaller bases of length $2 \varepsilon^{3}$ of the trapezoids, and the opposite sides of the rectangles coincide with the larger bases of the trapezoids. If the obtained figures (rectangles, sectors and trapezoids) do not mutually intersect, then their union is an $\varepsilon$-channel. It will be called the channel generated by the given $\varepsilon$-contour. 


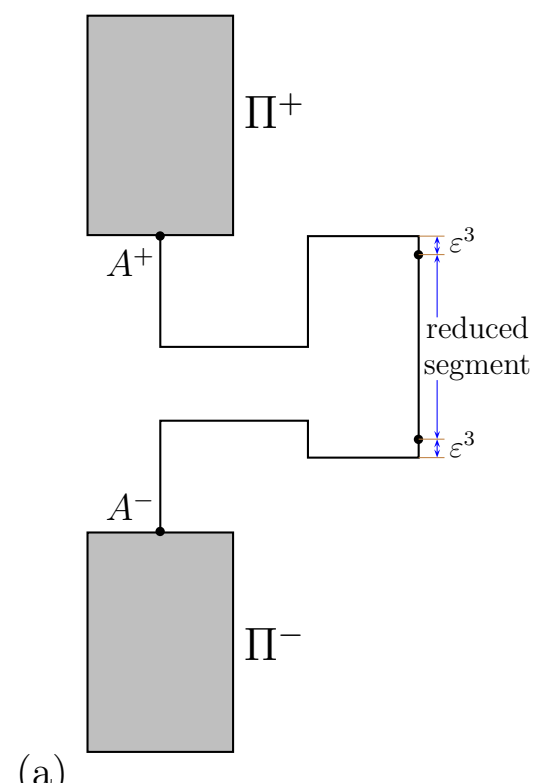

(a)

Figure 7: An $\varepsilon$-contour (a) and the channel generated by this contour (b).

\section{Proof of theorem 2}

Let the plane bodies $C_{1}$ and $C_{2}$ be given. Without loss of generality we assume that $v=(0,-1)$. The proof of Theorem 2 amounts to constructing a family of bodies $B_{\varepsilon}$, $C_{1} \subset B_{\varepsilon} \subset C_{2}$ with resistance going to zero, $\lim _{\varepsilon \rightarrow 0} R_{v}\left(B_{\varepsilon}\right)=0$. In what follows we will write $R$ instead of $R_{v}$, omitting the subscript $v$.

It suffices to provide the family $B_{\varepsilon}$ in the special case where

$$
\operatorname{dist}\left(\partial C_{1}, \partial C_{2}\right)>4 \sqrt{2} .
$$

Indeed, in the general case take $k>0$ large enough so that $\operatorname{dist}\left(\partial\left(k C_{1}\right), \partial\left(k C_{2}\right)\right)>4 \sqrt{2}$ and find a family $\tilde{B}_{\varepsilon}, k C_{1} \subset \tilde{B}_{\varepsilon} \subset k C_{2}$ such that $\lim _{\varepsilon \rightarrow 0} R\left(\tilde{B}_{\varepsilon}\right)=0$. Then the family $B_{\varepsilon}=k^{-1} \tilde{B}_{\varepsilon}$ satisfies the required relations $C_{1} \subset B_{\varepsilon} \subset C_{2}$ and $R\left(B_{\varepsilon}\right)=k^{-1} R\left(\tilde{B}_{\varepsilon}\right) \rightarrow 0$ as $\varepsilon \rightarrow 0$. Thus, the general case is reduced to the special case (3).

Consider the partition of $\mathbb{R}^{2}$ into (closed) squares of size $2 \times 2$ with vertices in $2 \mathbb{Z} \times 2 \mathbb{Z}$ and denote by $D$ the union of squares contained in the interior of $C_{2}$. One easily sees that $C_{1} \subset D$. The squares of the partition that are contained in $D$ and have nonempty intersection with $\partial D$ will be called boundary squares. The boundary squares do not intersect with $C_{1}$.

In Figure 8, $C_{1}$ and $C_{2}$ are bounded by black closed curves, and $D$ is bounded by the thick polygonal line. The boundary squares are situated between the thick and thin black solid polygonal lines. 
For future convenience we will use the small parameter $\varepsilon$ of the form $\varepsilon=1 /(2 n+1)$, where $n$ is a positive integer, and impose the restriction $\varepsilon<\operatorname{dist}\left(D, \partial C_{2}\right)$. Denote by $l=l(D)=\max \left\{x_{1}-y_{1}:\left(x_{1}, x_{2}\right),\left(y_{1}, y_{2}\right) \in D\right\}$ the width of $D$ and impose one more restriction $\varepsilon<1 / l$.

Denote by $\partial_{+} D$ and $\partial_{-} D$ the upper and lower parts of the boundary $\partial D$, that is, the intersection of $\partial D$ with the union of upper (lower) sides of the squares forming $D$. Introduce the metric $\bar{d}$ in $\mathbb{R}^{2}$ by $\bar{d}(x, y)=\max \left\{\left|x_{1}-y_{1}\right|,\left|x_{2}-y_{2}\right|\right\}$, where $x=\left(x_{1}, x_{2}\right)$ and $y=\left(y_{1}, y_{2}\right)$. In this metric a ball of radius $r$ is a square of size $2 r \times 2 r$ with vertical and horizontal sides.

Take $d_{i}=(2 i-1) \varepsilon^{3}, N=l /\left(2 \varepsilon^{2}\right)$, and denote by $L_{i}$ the set of points $x \in D$ such that $\bar{d}(x, \partial D)=d_{i}, i=1, \ldots, N$. The curve $L_{i}$ will be called the ith level line. Due to the choice of $\varepsilon$ one always has $d_{i}<1$, so each curve $L_{i}$ is contained in the union of boundary squares, and therefore, does not intersect $C_{1}$. The curves $L_{i}$ are closed, do not have selfintersections, and are composed of vertical and horizontal segments. Let us divide each level line $L_{i}$ into two curves by two points with maximal and minimal $x_{1}$-coordinates; then the $x_{1}$-coordinate will monotonically change along each of these curves. Finally, the $x_{1}$-coordinate of each vertical segment forming $L_{i}$ differs by $(2 i-1) \varepsilon^{3}$ from an integer, hence the difference of $x_{1}$-coordinates of any two vertical segments belonging to any two level lines is a multiple of $2 \varepsilon^{3}$. The same is valid for the $x_{2}$-coordinate. These observations imply that the length of each segment in each level line is more or equal than $2 \varepsilon^{3}$.

Divide the upper boundary, $\partial_{+} D$, into segments of length $2 \varepsilon^{2}$. The number of these segments is $N$, and the upper side of each square forming $\partial_{+} D$ contains exactly $\varepsilon^{-2}$ segments (recall that this number is integer). Denote the segments, from right to left (that is, from the larger to the smaller $x_{1}$-coordinate), by $I_{1}^{+}, \ldots, I_{N}^{+}$, and construct the rectangles $\Pi_{1}^{+}, \ldots, \Pi_{N}^{+}$of height $\varepsilon-\varepsilon^{2}$ resting on these segments; that is, $I_{i}^{+}$is the lower side of $\Pi_{i}^{+}$. Similarly, divide the lower boundary, $\partial_{-} D$, into segments of the same length, enumerate them from right to left, $I_{1}^{-}, \ldots, I_{N}^{-}$, and take the rectangles $\Pi_{1}^{-}, \ldots, \Pi_{N}^{-}$of the same height $\varepsilon-\varepsilon^{2}$ resting on these segments; that is, each segment $I_{i}^{-}$is the upper side of $\Pi_{i}^{-}$. The rectangles $\Pi_{i}^{+}$and $\Pi_{i}^{-}$, corresponding to three different values of $i$, are shown in Fig. 8.

All the rectangles $\Pi_{i}^{ \pm}$are contained in $C_{2} \backslash D$. Denote by $A_{i}^{+}$the midpoint of the segment $I_{i}^{+}$, and by $A_{i}^{-}$, the midpoint of $I_{i}^{-}$. The $x_{1}$-coordinate of both $A_{i}^{+}$and $A_{i}^{-}$ equals

$$
x_{1}\left(A_{i}^{+}\right)=\varepsilon^{2}(1+2 N-2 i) .
$$

Denote by $A_{i}^{+} B_{i}^{+}$and $A_{i}^{-} B_{i}^{-}$the connected components of the set

$$
\left\{x: x_{1}=x_{1}\left(A_{i}^{+}\right), \bar{d}(x, \partial D) \leq d_{i}\right\}
$$

containing the points $A_{i}^{+}$and $A_{i}^{-}$, respectively. Both of them are vertical segments, and either both coincide, or not coincide with $A_{i}^{+} A_{i}^{-}$. In the latter case one has $\bar{d}\left(B_{i}^{+}, \partial D\right)=$ $\bar{d}\left(B_{i}^{-}, \partial D\right)=d_{i}$. 


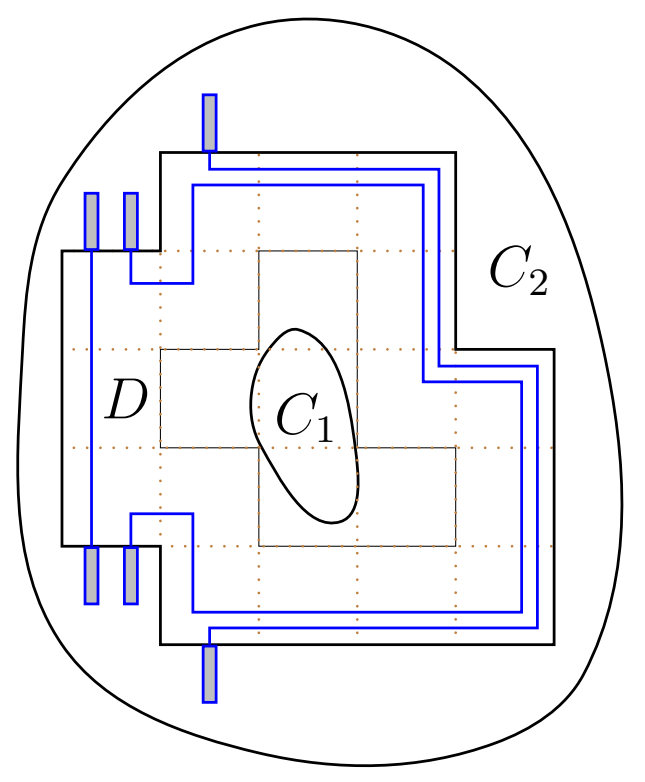

Figure 8: The construction of a built-in channel system: the $\varepsilon$-contours for three values of $i$ are shown blue.

Define the broken line $\Gamma_{i}$ as follows. If both $A_{i}^{+} B_{i}^{+}$and $A_{i}^{-} B_{i}^{-}$coincide with the segment $A_{i}^{+} A_{i}^{-}$, then $\Gamma_{i}$ is identical to this segment. Otherwise $\Gamma_{i}$ is the union of the segments $A_{i}^{+} B_{i}^{+}$and $A_{i}^{-} B_{i}^{-}$and the part of the curve $L_{i}$ contained in the half-plane $x_{1}>x_{1}\left(A_{i}^{+}\right)$. In the latter case the length of each of the segments $A_{i}^{+} B_{i}^{+}$and $A_{i}^{-} B_{i}^{-}$is more or equal than $\varepsilon^{3}$.

By formula (4) and by the choice of $\varepsilon$, the value $x_{1}\left(A_{i}^{+}\right)-\varepsilon^{3}$ is a multiple of $2 \varepsilon^{3}$. Besides, it has been already established that each vertical segment of the broken line $L_{i}$ also has this property: denoting by $x_{1}$ the first coordinate of the segment, we have that $x_{1}-\varepsilon^{3}$ is a multiple of $2 \varepsilon^{3}$. Thus, the distance from each of the vertical segments $A_{i}^{+} B_{i}^{+}$, $A_{i}^{-} B_{i}^{-}$to the nearest vertical segment of $L_{i}$ is a multiple of $2 \varepsilon^{3}$. This implies that the lengths of the first and the last horizontal segments of $\Gamma_{i}$ are more or equal than $2 \varepsilon^{3}$. The other intermediate segments of $\Gamma_{i}$ are at the same time segments of $L_{i}$, and therefore, also have lengths more or equal than $2 \varepsilon^{3}$.

Thus, the broken line $\Gamma_{i}$ is composed of vertical and horizontal segments, the lengths of the initial and the final segments are more or equal than $\varepsilon^{3}$, and the lengths of the other segments are more or equal than $2 \varepsilon^{3}$. In particular, this broken line may coincide with a single vertical segment. It starts at the point $A_{i}^{+}$and finishes at the point $A_{i}^{-}$and does not have points of self-intersection. Therefore $\Gamma_{i}$ is an $\varepsilon$-contour joining the rectangles 
$\Pi_{i}^{+}$and $\Pi_{i}^{-}$. The $\bar{d}$-distance between different curves $\Gamma_{i}$ and $\Gamma_{j}$ is at least $2 \varepsilon^{3}$,

$$
\bar{d}\left(\Gamma_{i}, \Gamma_{j}\right) \geq 2 \varepsilon^{3} \quad \text { for } i \neq j .
$$

Fix $i$ and consider the rectangles and sectors generated by the reduced segments and vertices of the broken line $\Gamma_{i}$. Notice that any triple of consecutive elements: a $v$-rectangle, a sector, and an $h$-rectangle, contains elements that do not intersect pairwise. On the other hand, if a pair of elements does not belong to such a triple, then the minimal union of the squares of the partition containing one element does not intersect the minimal union of the squares containing the other element. Therefore all the elements do not mutually intersect; hence these elements, jointly with the trapezoids $V_{i}^{+} \subset \Pi_{i}^{+}$and $V_{i}^{-} \subset \Pi_{i}^{-}$ generated by the rectangles $\Pi_{i}^{+}$and $\Pi_{i}^{-}$, form a channel; let it be denoted by $\tilde{K}_{i}$. We assume that the channel is open, that is, does not intersect its boundary.

Now let us show that the channels defined above do not mutually intersect. Let $K_{i}^{\prime}$ be the union of the rectangles (or the rectangle), generated by the segments $A_{i}^{+} B_{i}^{+}, A_{i}^{-} B_{i}^{-}$, and the adjacent sectors. Let $K_{i}^{\prime \prime}$ be the union of the other sets generated by the broken line $\Gamma_{i}$ and forming the channel. One obviously has

$$
\tilde{K}_{i}=K_{i}^{\prime} \cup K_{i}^{\prime \prime} \cup V_{i}^{+} \cup V_{i}^{-} \subset K_{i}^{\prime} \cup K_{i}^{\prime \prime} \cup \Pi_{i}^{+} \cup \Pi_{i}^{-} .
$$

We have $K_{i}^{\prime} \subset\left\{x: \bar{d}(x, \partial D) \leq 2 i \varepsilon^{3}\right\}$ and $K_{i}^{\prime \prime} \subset\left\{x:(2 i-2) \varepsilon^{3}<\bar{d}(x, \partial D)<2 i \varepsilon^{3}\right\}$; hence

$$
K_{i}^{\prime \prime} \cap K_{j}^{\prime \prime}=\emptyset \text { for } i \neq j \text { and } K_{i}^{\prime} \cap K_{j}^{\prime \prime}=\emptyset \text { for } i<j .
$$

Further, $K_{i}^{\prime} \subset\left\{x: x_{1}\left(A_{i}^{+}\right)-\varepsilon^{3}<x_{1}<x_{1}\left(A_{i}^{+}\right)+\varepsilon^{3}\right\}$ and $K_{i}^{\prime \prime} \subset\left\{x: x_{1}>x_{1}\left(A_{i}^{+}\right)+\varepsilon^{3}\right\}$, hence

$$
K_{i}^{\prime} \cap K_{j}^{\prime}=\emptyset \quad \text { and } \quad K_{i}^{\prime \prime} \cap K_{j}^{\prime}=\emptyset \text { for } i<j .
$$

Finally, both the sets $\Pi_{i}^{+} \cup \Pi_{i}^{-}$and $\Pi_{j}^{+} \cup \Pi_{j}^{-}, i \neq j$ do not intersect with $D$ and have non-intersecting projections on the horizonal axis; therefore

$$
\left(\Pi_{i}^{+} \cup \Pi_{i}^{-}\right) \cap\left(\Pi_{j}^{+} \cup \Pi_{j}^{-}\right)=\emptyset \quad \text { and } \quad\left(\Pi_{i}^{+} \cup \Pi_{i}^{-}\right) \cap\left(K_{j}^{\prime} \cup K_{j}^{\prime \prime}\right)=\emptyset \text { for } i \neq j .
$$

The relations (5)-(8) imply that the channels $\tilde{K}_{i}, i=1, \ldots, N$ do not mutually intersect. Moreover, the union of the front sides of these channels is a horizontal segment of length $l$ shielding the vertical flow of particles incident on $D$.

Denote

$$
\tilde{B}_{\varepsilon}=D \cup\left(\cup_{i} \Pi_{i}^{+}\right) \cup\left(\cup_{i} \Pi_{i}^{-}\right) \backslash\left(\cup_{i} \tilde{K}_{i}\right)
$$

Thus, the set $\tilde{B}_{\varepsilon}$ is obtained by adding all the rectangles $\Pi_{i}^{ \pm}$to the set $D$ and then subtracting all the channels $\tilde{K}_{i}$. A particle, incident on $D$ with the initial velocity $v=$ $(0,-1)$, intersects the front side of a channel, passes through the channel in the positive 
direction, then intersects its rear side and further moves with a velocity $v^{+}=v+O(\varepsilon)$. Therefore the resistance of $\tilde{B}_{\varepsilon}$ equals

$$
R\left(\tilde{B}_{\varepsilon}\right)=O(\varepsilon), \quad \varepsilon \rightarrow 0 .
$$

Notice that, roughly speaking, the set $\tilde{B}_{\varepsilon}$ is not a body, since it is locally onedimensional. Indeed, the intersection of $\tilde{B}_{\varepsilon}$ with a neighborhood of a point on the common boundary of neighbor $v$ - or $h$-rectangles is a rectilinear interval. In order to improve the construction, replace the $\varepsilon$-channels $\tilde{K}_{i}$ in formula (9) with $\varepsilon^{\prime}$-channels $K_{i}$ contained in $\tilde{K}_{i}$, with $\varepsilon^{\prime}=\varepsilon+O\left(\varepsilon^{2}\right)<\varepsilon$. (We additionally require that the lateral boundaries of $\tilde{K}_{i}$ and $K_{i}$ are disjoint.) The resulting set $B_{\varepsilon}=D \cup\left(\cup \Pi_{i}^{+}\right) \cup\left(\cup \Pi_{i}^{-}\right) \backslash\left(\cup K_{i}\right)$ is a true body, and it also satisfies the relation $R\left(B_{\varepsilon}\right)=O(\varepsilon), \varepsilon \rightarrow 0$ and the inclusion $C_{1} \subset B_{\varepsilon} \subset C_{2}$. The theorem is proved.

\section{Proof of theorem 1}

Fix the bodies $C_{1}$ and $C_{2}$ and assume, without loss of generality, that $v=(0,0,-1)$. Like in the previous section, we construct here a family of connected bodies $B_{\varepsilon}, C_{1} \subset B_{\varepsilon} \subset C_{2}$ with vanishing resistance, $\lim _{\varepsilon \rightarrow 0} R\left(B_{\varepsilon}\right)=0$.

A typical body of the family is sandwich-shaped: it is the union of several thin sheets of two kinds: "sheets of small resistance" and "solid sheets". These two kinds of sheets alternate in the sandwich. The plane of the sheets is parallel to $v$. The sheets of small resistance are constructed with the use of Theorem 2 proved in the previous section. The solid sheets are much thinner than the sheets of small resistance and "glue them together", so that the resulting body is connected.

Let us proceed to the description of the construction. For a convex body $C \subset \mathbb{R}^{3}$ denote

$$
C^{t}=\left\{\left(x_{2}, x_{3}\right):\left(t, x_{2}, x_{3}\right) \in C\right\} .
$$

In other words, $C^{t}$ is the projection of the cross section $C \cap\left\{x_{1}=t\right\}$ on the plane $\mathbb{R}_{\left\{x_{2}, x_{3}\right\}}^{2}$. Further, for a set $A \subset \mathbb{R}$ define the sets

$$
\underline{C}^{A}=\cap_{t \in A} C^{t} \quad \text { and } \quad \bar{C}^{A}=\operatorname{Conv}\left(\cup_{t \in A} C^{t}\right),
$$

where Conv means convex hull. One easily sees that for any $t \in A$ holds

$$
\bar{C}^{A} \subset C^{t} \subset \underline{C}^{A} .
$$

Define the set

$$
I=\left\{t: C_{1}^{t} \neq \emptyset\right\}
$$

that is, $I$ is the projection of $C_{1}$ on $\mathbb{R}_{\left\{x_{1}\right\}}$. Without loss of generality assume that the sets $C_{1}$ and $C_{2}$ are open. Then $I$ is a bounded open interval, $I=(a, b)$. 
Further, one has $C_{1}^{t} \subset C_{2}^{t}$. Moreover, for any $t \in I$ there exists an open interval $\mathcal{U}_{t}$ containing $t$ such that

$$
\bar{C}_{1}^{\mathcal{U}_{t}} \subset \underline{C}_{2}^{\mathcal{U}_{t}} \quad \text { and } \quad \partial\left(\bar{C}_{1}^{\mathcal{U}_{t}}\right) \cap \partial\left(\underline{C}_{2}^{\mathcal{U}_{t}}\right)=\emptyset .
$$

Fix $\varepsilon>0$ and choose a finite subset of intervals $\mathcal{U}_{t_{i}}$ covering the segment $[a+\varepsilon, b-\varepsilon]$. Next choose disjoint intervals $I_{i}^{\varepsilon} \subset \mathcal{U}_{t_{i}}$ such that

$$
\cup_{i} I_{i}^{\varepsilon}=[a+\varepsilon, b-\varepsilon] .
$$

Due to the choice of these intervals, for each $i$ one has

$$
\bar{C}_{1}^{I_{i}} \subset \underline{C}_{2}^{I_{i}} \quad \text { and } \quad \partial\left(\bar{C}_{1}^{I_{i}}\right) \cap \partial\left(\underline{C}_{2}^{I_{i}}\right)=\emptyset .
$$

Define

$$
C(\varepsilon)=C_{2} \cap\left\{x_{1} \in(a, a+\varepsilon) \cup(b-\varepsilon, b)\right\}
$$

and denote by $C_{x_{1}, x_{2}}(\varepsilon)$ the projection of $C(\varepsilon)$ on the plane $\mathbb{R}_{\left\{x_{1}, x_{2}\right\}}^{2}$. More precisely, one has

$$
C_{x_{1}, x_{2}}(\varepsilon)=\left\{(t, \tau): t \in(a, a+\varepsilon) \cup(b-\varepsilon, b) \text { and } C_{2}^{t} \cap\left\{x_{2}=\tau\right\} \neq \emptyset\right\} .
$$

Let $a_{\varepsilon}$ be the area of $C_{x_{1}, x_{2}}(\varepsilon)$; one has $\lim _{\varepsilon \rightarrow 0} a_{\varepsilon}=0$. The resistance of $C(\varepsilon)$ can be estimated as

$$
|R(C(\varepsilon))| \leq 2 a_{\varepsilon} .
$$

Using Theorem 2, choose plane sets $B_{i}^{\varepsilon}$ such that

$$
\bar{C}_{1}^{I_{i}} \subset B_{i}^{\varepsilon} \subset \underline{C}_{2}^{I_{i}} \quad \text { and } \quad\left|R\left(B_{i}^{\varepsilon}\right)\right|<\varepsilon .
$$

Then the resistance of the three-dimensional set $I_{i} \times B_{i}^{\varepsilon}$ can be estimated as

$$
\left|R\left(I_{i} \times B_{i}^{\varepsilon}\right)\right|=\left|I_{i}\right| \cdot\left|R\left(B_{i}^{\varepsilon}\right)\right|<\varepsilon\left|I_{i}\right|,
$$

where $|I|$ means the length of the interval $I$. Define the set

$$
\tilde{B}_{\varepsilon}=C(\varepsilon) \cup\left(\cup_{i} I_{i} \times B_{i}^{\varepsilon}\right) .
$$

Using the first relation in (12) and the definition of $C(\varepsilon)$, one concludes that

$$
C_{1} \subset \tilde{B}_{\varepsilon} \subset C_{2} .
$$

Consider a particle incident on the body $\tilde{B}_{\varepsilon}$. If its $x_{1}$-coordinate belongs to $(a, a+$ $\varepsilon) \cup(b-\varepsilon, b)$, then it makes a single reflection from the body at a point of $C(\varepsilon)$. If the $x_{1}$-coordinate belongs to an interval $I_{i}$, then the particle makes several reflections at 
points of $I_{i} \times B_{i}^{\varepsilon}$ and never hits any other subset constituting the body $\tilde{B}_{\varepsilon}$. Therefore the resistance of $\tilde{B}_{\varepsilon}$ is the sum of resistances of its subsets,

$$
R\left(\tilde{B}_{\varepsilon}\right)=R(C(\varepsilon))+\sum_{i} R\left(I_{i} \times B_{i}^{\varepsilon}\right)
$$

Using (11) and (13), one gets the estimate

$$
\left|R\left(\tilde{B}_{\varepsilon}\right)\right| \leq 2 a_{\varepsilon}+\varepsilon(b-a)
$$

that is, $\lim _{\varepsilon \rightarrow 0} R\left(\tilde{B}_{\varepsilon}\right)=0$.

However, the set $\tilde{B}_{\varepsilon}$ is not connected. Let us therefore modify it in the following way. Take an open set $J_{\varepsilon} \subset \mathbb{R}$ and require that it is the disjoint union of open intervals of total length less than $\varepsilon$ and contains the endpoints of all the intervals $I_{i}$, that is,

$$
\cup_{i} \partial I_{i} \subset J_{\varepsilon} \quad \text { and } \quad\left|J_{\varepsilon}\right|<\varepsilon
$$

Define

$$
D(\varepsilon)=C_{2} \cap\left\{x_{1} \in J_{\varepsilon}\right\}
$$

Then the body

$$
B_{\varepsilon}=\tilde{B}_{\varepsilon} \cup D(\varepsilon)
$$

is connected and satisfies the relations

$$
C_{1} \subset B_{\varepsilon} \subset C_{2} \quad \text { and } \quad \lim _{\varepsilon \rightarrow 0} R\left(B_{\varepsilon}\right)=0
$$

Theorem 1 is proved.

\section{Acknowledgements}

This work was supported by the Center for Research and Development in Mathematics and Applications (CIDMA) from the "Fundação para a Ciência e a Tecnologia" (FCT), cofinanced by the European Community Fund FEDER/POCTI, and by the FCT research project PTDC/MAT/113470/2009.

\section{References}

[1] M. Belloni and B. Kawohl. A paper of Legendre revisited. Forum Math. 9, 655-668 (1997).

[2] M. Belloni and A. Wagner. Newtons problem of minimal resistance in the class of bodies with prescribed volume. J. Convex Anal. 10, 491500 (2003). 
[3] F. Brock, V. Ferone, B. Kawohl. A symmetry problem in the calculus of variations. Calc. Var. 4, 593-599 (1996).

[4] D. Bucur and G. Buttazzo. Variational Methods in Shape Optimization Problems. Birkhäuser (2005).

[5] G. Buttazzo, V. Ferone, B. Kawohl. Minimum problems over sets of concave functions and related questions. Math. Nachr. 173, 71-89 (1995).

[6] G. Buttazzo, P. Guasoni, Shape optimization problems over classes of convex domains, J. Convex Anal. 4, No.2, 343-351 (1997).

[7] G. Buttazzo, B. Kawohl. On Newton's problem of minimal resistance. Math. Intell. 15, 7-12 (1993).

[8] M. Comte, T. Lachand-Robert. Newton's problem of the body of minimal resistance under a single-impact assumption. Calc. Var. Partial Differ. Equ. 12, 173-211 (2001).

[9] M. Comte, T. Lachand-Robert. Existence of minimizers for Newton's problem of the body of minimal resistance under a single-impact assumption. J. Anal. Math. 83, 313-335 (2001).

[10] M. Comte and T. Lachand-Robert. Functions and domains having minimal resistance under a single-impact assumption. SIAM J. Math. Anal. 34 (2002), 101-120.

[11] T. Lachand-Robert, M. A. Peletier. Newton's problem of the body of minimal resistance in the class of convex developable functions. Math. Nachr. 226, 153-176 (2001).

[12] T. Lachand-Robert, M. A. Peletier. An example of non-convex minimization and an application to Newton's problem of the body of least resistance. Ann. Inst. H. Poincaré, Anal. Non Lin. 18, 179-198 (2001).

[13] I. Newton, Philosophiae Naturalis Principia Mathematica (London: Streater). 1687.

[14] A. Plakhov. Billiard scattering on rough sets: Two-dimensional case. SIAM J. Math. Anal. 40, 2155-2178 (2009).

[15] A. Plakhov. Billiards and two-dimensional problems of optimal resistance. Arch. Ration. Mech. Anal. 194, 349-382 (2009).

[16] A. Plakhov. Scattering in billiards and problems of Newtonian aerodynamics. Russ. Math. Surv. 64, 873938 (2009). 